\title{
High Resilient Messaging Service for Microservice Architecture
}

\author{
Sanjana G B \\ Department of Computer Science and Engineering \\ R.V. College of Engineering \\ Bengaluru, Karnataka, India-560059
}

\author{
Prof. Girish Rao Salanke N S \\ Department of Computer Science and Engineering \\ R.V. College of Engineering \\ Bengaluru, Karnataka, India-560059
}

\begin{abstract}
Fundamental structure of a software system is called software architecture. Software architectures are helpful in systematic software development. One such software architecture is microservice architecture. This architecture breaks down the entire software system into smaller components each of which work independently. The microservice architecture is being widely adapted in software industries due to its reliability, scalability and easier maintenance. But breaking down a system into smaller, independent components that use different tech stacks and message formats gives rise to complex communication between the components. This paper proposes a new messaging service that allows communication between services with ease. The new messaging service uses distributed streaming platforms like Apache Kafka to decouple the messaging between services. It also uses Apache Camel to provide functionalities such as message schema transformation and schema validation; these functionalities allow the services to communicate with each other with only a few lines of code. The result is a new state of the art messaging service that can be easily integrated with producer and consumer services. The paper also discusses where the new messaging service is more suitable. The performance metric used here is the number of additional lines of code required on the producer or consumer side for using this messaging service. The results are, almost thirty percent to sixty percent reduction of code required for integration on the producer side and nine percent to forty percent reduction of code required for integration on the consumer side.
\end{abstract}

Index Terms-Microservice, Interprocess communication, Apache Kafka, Apache Camel, Jolt.

\section{INTRODUCTION}

Microservice is a relatively new architecture that is becoming dominant in service oriented software companies [4]. The trends in microservice architecture over the years is shown in Fig. 1. Microservice allows a system to be divided into small services that are built to perform a cohesive business function. This has a number of benefits such as enhanced scalability, easy maintainability, and autonomy between services.

One of the key challenges of microservices is the complexity introduced by it. Each service operates independently and has a different technology stack [5]. This leads to complexity when one service wants to communicate with another. Hence deciding how to allow communication between services is an important task in designing a microservice architecture.

The communication method should be such that it does not defeat the whole purpose of microservices. It should

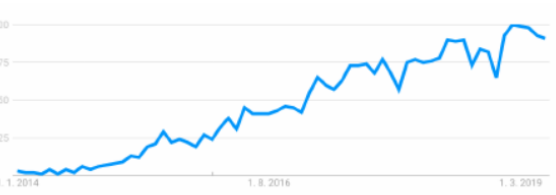

Figure 1: Google search trends for microservices

Fig. 1. Google search trends in microservices [2].

allow efficient communication between microservices while maintaining loose coupling between the services. It should also allow enhanced scalability, easy maintainability, and autonomy between services. This type of communication is also called Interprocess communication (IPC).

The goal of this paper is to study two most commonly used IPC, synchronous and asynchronous, and create a new design to overcome their pitfalls. The paper introduces a new messaging service through a design that overcomes the drawbacks of the existing interprocess communication.

The design used to create an efficient messaging service includes using asynchronous streaming platforms such as Kafka [6] and enterprise integration patterns provided by integration frameworks such as Camel [3]. Kafka allows communication between services without breaking the autonomous nature of the services. Camel is a lightweight integration framework. It allows the routing of messages from the producer services to appropriate consumer service using enterprise integration patterns. Since Camel can communicate with Kafka, it allows the design of an efficient messaging service that is an amalgam of integration patterns and asynchronous IPC.

The remaining content is organised as follows. Section 2 gives a brief overview of the synchronous and asynchronous Interprocess communication and their drawbacks. Section 3 talks about related research work. Section 4 discusses the proposed solution and a detailed implementation and the working of the proposed messaging service. Section 5 discusses the results, advantages, disadvantages and applications of the new design. Section 6 concludes the paper. The final section speaks about how the proposed design can be improved in future work. 
International Journal of Applied Engineering Research ISSN 0973-4562 Volume 16, Number 5 (2021) pp. 357-361

(c) Research India Publications. https://dx.doi.org/10.37622/IJAER/16.5.2021.357-361

\section{INTERPROCESS COMMUNICATION IN MICROSERVICE}

\section{A. Synchronous Communication}

Synchronous communication uses a request/response style of interaction. In this a microservice requests service from another microservice and blocks itself till the request is completed and a response is sent to the requester service.

The most commonly used request/response style of interactions for microservices are HTTP-based REST application programming interface (API) and google Remote Procedure call (gRPC). Because of its simplicity and easy availability HTTP-based REST API is more commonly used.

In a system that uses REST API for its IPC communication, each service has its own web-server running on a specific port, and each service exposes a set of endpoints to allow the interactions with other microservices. The service API has two parts: the resource and the verb. The resource can be accessed using Uniform Resource Identifier (URI) and the verb tells about the HTTP method to be performed. Fig. 2 depicts synchronous HTTP-based REST API.

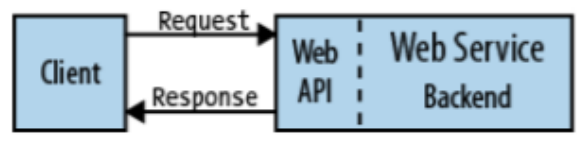

Fig. 2. Synchronous HTTP-based REST [5].

The main drawback of this style of interaction is that the requesting service blocks until it receives a response. This in turn defeats the purpose of microservices.

\section{B. Asynchronous Communication}

Asynchronous communication uses a publish/subscribe style of interaction. In this a microservice (producer) publishes a message to a message broker and continues its other tasks. Then another service (consumer) takes the message from the message broker and processes it and places it back on the message broker.

The most commonly used protocol which is messaging based is Advanced Message Queuing Protocol (AMQP), some message brokers commonly used are RabbitMQ and Apache Kafka. Because of its ability to provide real-time data processing Apache Kafka is most commonly used. Fig. 3 depicts asynchronous publish/subscribe messaging style.

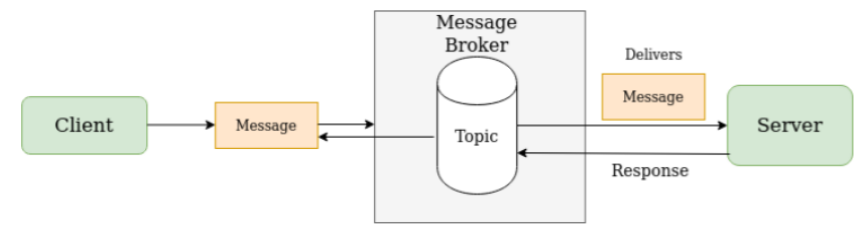

Fig. 3. Asynchronous publish/subscribe messaging [5].

The drawback of this method is that a system requires additional lines of code to connect to the message brokers.
Also when the schema of the message from the producer is different from the schema of the message required by the consumer, the message has to be transformed. This requires a series of operations that has to be coded on either the producer or consumer side. This in turn causes an overhead of adding code to the services. The message brokers also do not ensure schema compatibility. Thus a messaging service which assures schema compatibility and reduces the amount of code to be added is necessary.

\section{Message Format}

Besides choosing the right IPC another important decision to make is choosing the message format. Message contains information that is processed by the microservices. There are various message formats available such as JavaScript Object Notation (JSON), Google Protocol Buffer, Apache Avro. For the purpose of this project JSON will be chosen as the standard message format. JSON is a human readable data format that is widely used in the industries [1]. It can be easily serialized and deserialized, almost hundred times faster than Extensible Markup Language (XML) [5].

\section{RELATED WORK}

In reference paper [5], research on interprocess communication in microservice architecture is carried out. Here an e-commerce scenario has been implemented to simulate microservice architecture. Synchronous and asynchronous style of communication is studied and compared for the e-commerce use case. It is observed that the performance of the system with asynchronous communication is better when the load is more, whereas the performance of the system with synchronous communication is slightly better when the load is less. Fig. 4 displays the results of the models in reference paper [5].

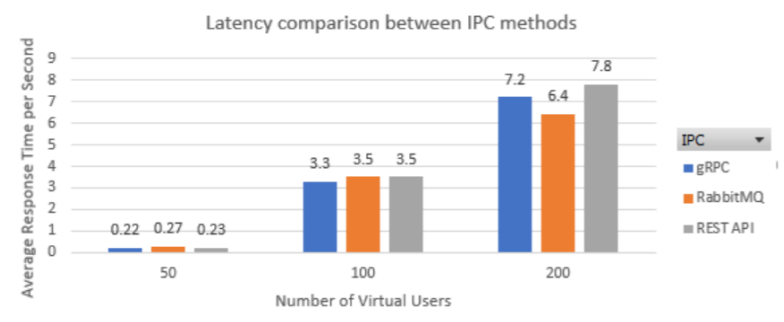

Fig. 4. Results obtained in reference paper [5].

In reference paper [2], study on various IPC technologies, such as synchronous REST and asynchronous Messaging Queue (MQ) is conducted and the performance of the technologies with varying message size is determined. One of the most interesting findings in this research is that up to fourteen Mega Byte (MB) of data, both REST and MQ perform the same. Above fourteen MB and upto fifty MB the performance of MQ is slightly better than REST. Only above fifty MB does performance of the two technologies vary by a large margin and MQ outperforms REST.

From these literature papers we can conclude that synchronous communication serves well when the load is less, 
International Journal of Applied Engineering Research ISSN 0973-4562 Volume 16, Number 5 (2021) pp. 357-361

(C) Research India Publications. https://dx.doi.org/10.37622/IJAER/16.5.2021.357-361

but asynchronous communication scales better as the load increases. These results are helpful in choosing the right IPC based on requirements such as message size, number of requests.

Reference paper [7] studies about data management in microservice architecture. Data management is very critical especially while migrating from monolithic to microservice architecture. In this literature they report about data management and how to improve the performance of the data source. They also use two use cases to illustrate their design and point out where their design will be useful. The approach used by them to design a messaging architecture has been helpful in coming up with the design in this paper.

But the major drawbacks of the above research is that none of the papers speak about additional lines of code required by a service to use the different types of IPC. This is an important evaluation metric during the modernization journey.

Thus creating a service that reduces the number of code required by one service to communicate with another service is the goal of the paper.

\section{Proposed Solution}

\section{A. Overview}

The solution proposed in this paper overcomes the drawback of synchronous communication using a distributed streaming platform Apache Kafka. Apache Kafka is a message broker that supports asynchronous communication based on publish/subscribe style.

Asynchronous messaging is chosen because it can scale easily at any load. Apache Kafka is chosen because of its ability to support real time data transmission. But this asynchronous communication still has major drawbacks such as lesser scalability due to requirement of changes in code to accommodate necessary configuration details. The changes in code of producer/consumer services that use Kafka for communication are also required for message schema transformations when the schema of the producer and consumer service is different, and for schema compatibility validations. These functionalities are not provided by Kafka.

The messaging service designed in this paper removes the above drawbacks by using integration frameworks like Apache Camel, schema registries and json-to-json transformers. The detailed implementation is discussed in the next sub-section.

\section{B. Implementation}

The messaging service allows creation of a Camel route, using appropriate enterprise integration patterns, so that a publisher service can send the messages to multiple consumer services.

The Camel route starts consuming from a Kafka topic created for this messaging service. Camel also allows transformation and validation of the consumed messages before routing the message to appropriate endpoints which are topics in Kafka. Fig. 6 gives an overview of the messaging service proposed in this paper.

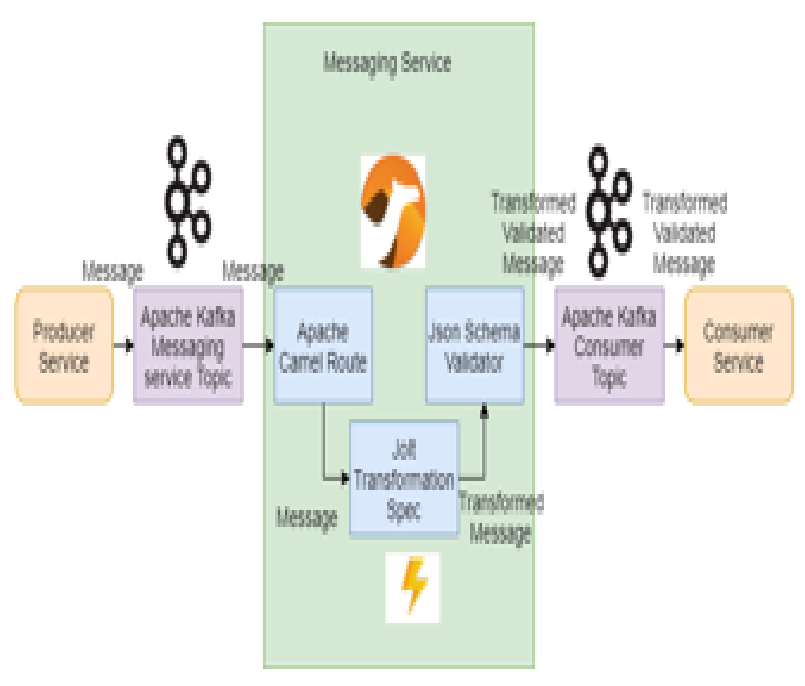

Fig. 5. Overview of the messaging service proposed in this paper.

The schema of JSON of producer and consumer service can be different. Then transformation of schema is required. The message schema transformation is supported using Jolt. Jolt is a Camel component that allows JSON processing using Jolt specifications. It allows JSON to JSON transformations.

Since the message on Kafka is binary it does not ensure protection against data corruption or schema change. To ensure integrity of schema, the schema has to be validated. The JSON schema validation is done using the json validator component provided by Camel. The reference schema is stored in a schema registry and is used for validating. Then the message is sent to appropriate endpoints.

Each of the end points in a Camel Route are Kafka topics. The consumer services subscribe to these topics and consume messages from the Kafka topics that they have subscribed to. This communication with Kafka is done using the Camel Kafka component that allows communication with Kafka message brokers.

This removes the burden of message transformation and validation from the producer/consumer services, hence reduces the number of lines of code required for IPC on the producer and the consumer services.

Hence the producer/consumer services can be easily integrated to use the messaging service proposed here.

\section{Working}

When a producer service wants to send messages to a single/multiple other consumer services, the engineering team that created the producer service can specify the Kafka topics 
International Journal of Applied Engineering Research ISSN 0973-4562 Volume 16, Number 5 (2021) pp. 357-361

(c) Research India Publications. https://dx.doi.org/10.37622/IJAER/16.5.2021.357-361

from which the consumer services consume, using the user interface (UI) provided by the messaging service in this paper.

They can also specify Jolt specifications for JSON to JSON transformation if required due to differences in JSON schema of the producer and the consumer service. This information is provided using the UI mentioned above.

On providing the above information a camel route is created. And details are stored in the database. The user can also provide reference schema to be stored in the schema registry and have to create appropriate consumer topics on Kafka message broker.

The producer service is now ready to send messages. It can send messages by publishing a message to the Kafka topic created for this messaging service, using Camel Kafka producer. All Camel routes in the messaging service consume from this topic.

The Camel then receives the message and does processing depending on Jolt specification provided while creating a route. These details are fetched from the database. It also validates the schema of the transformed message against the reference schema in schema registry. On success the messages are published to the Kafka topics specified on the Camel route's endpoints.

The consumer services can then consume the message from these topics to which they have subscribed.

The working of the messaging service is explained with the help of flowchart (Fig. 7).

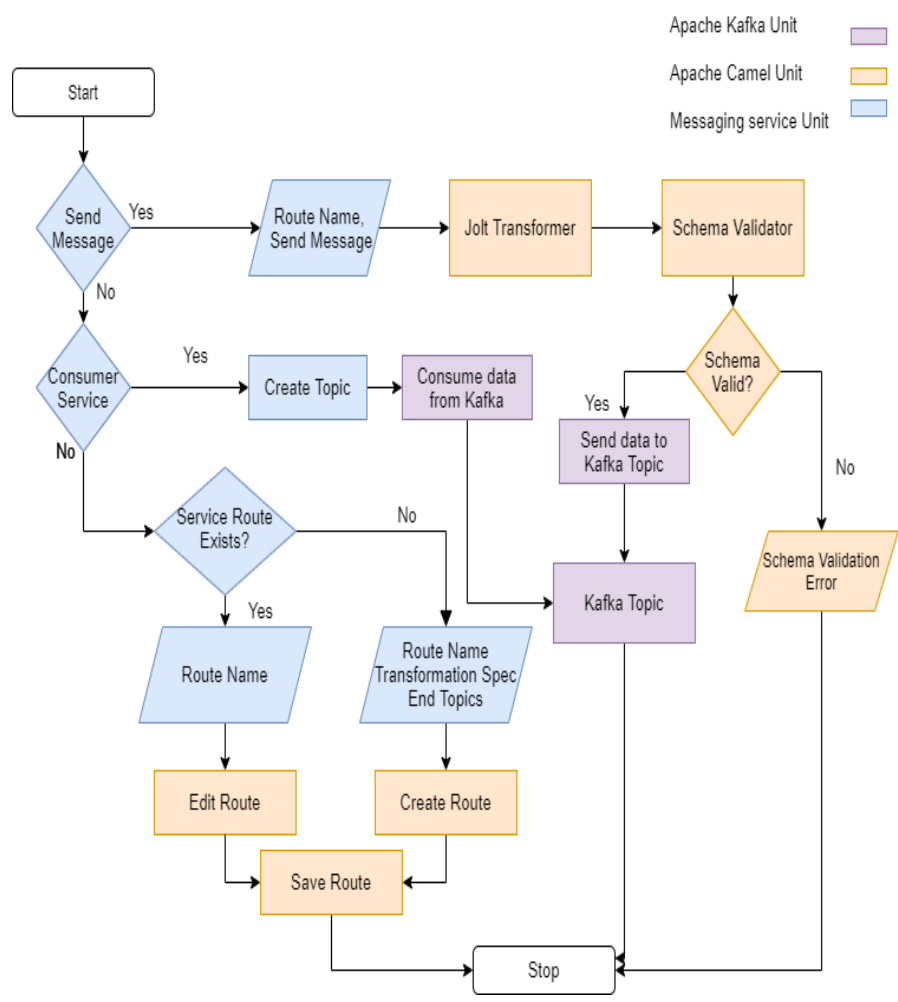

Fig. 6. Flowchart depicting the working of the service.

\section{RESUlts}

Most of the papers in literature survey evaluate a messaging service based on request/response time and the size of the message. The messaging model in this paper performs on par with the models in other research papers in terms of request/response time.

But another important factor to be considered while creating a messaging service is the ease with which the services can use the messaging service. For a service the number of lines of code that has to be added in order to use the messaging service determines the ease with which it can be used. Hence the evaluation metric chosen here is the additional lines of code required for each service, producer and consumer, to use this messaging.

Let us study the results of different cases:

Case 1 : Service A sends messages to Service B and Service $\mathrm{C}$ with transformation of messages. When the messaging service in this paper is not used an assumption that transformations and validations are done on the producer side is made. TABLE 1 represents the performance results of the messaging service proposed in this paper for Case 1.

TABLE I

TABLE FOR CASE 1 RESULTS

\begin{tabular}{|c|c|c|c|}
\hline \multirow{2}{*}{$\begin{array}{c}\text { Type of } \\
\text { Messaging Service }\end{array}$} & \multicolumn{3}{|c|}{ Additional Lines of Code } \\
\cline { 2 - 4 } & Service A & Service B & Service C \\
\hline $\begin{array}{l}\text { Asynchronous with Camel } \\
\text { messaging service }\end{array}$ & 21 & 29 & 29 \\
\hline $\begin{array}{l}\text { Asynchronous without Camel } \\
\text { messaging service }\end{array}$ & 56 & 32 & 32 \\
\hline
\end{tabular}

Case 2 : Service A sends messages to Service B and Service $\mathrm{C}$ with transformation of messages. When the messaging service in this paper is not used an assumption that transformations and validations are done on the consumer side is made. TABLE 2 represents the performance results of the messaging service proposed in this paper for Case 2.

TABLE II

TABLE FOR CASE 2 RESULTS

\begin{tabular}{|l|c|c|c|}
\hline \multirow{2}{*}{$\begin{array}{c}\text { Type of } \\
\text { Messaging Service }\end{array}$} & \multicolumn{3}{|c|}{ Additional Lines of Code } \\
\cline { 2 - 4 } & Service A & Service B & Service C \\
\hline $\begin{array}{l}\text { Asynchronous with Camel } \\
\text { messaging service }\end{array}$ & 21 & 29 & 29 \\
\hline $\begin{array}{l}\text { Asynchronous without Camel } \\
\text { messaging service }\end{array}$ & 30 & 42 & 42 \\
\hline
\end{tabular}

Case 3 : Service A sends a message to Service B and Service $\mathrm{C}$ without transformation of message. TABLE 3 represents the performance results of the messaging service proposed in this paper for Case 3.

From the above results it can be concluded that when there are transformations and validations required during communication, the messaging service proposed in the paper reduces the overall lines of code by almost $34 \%$, on the producer side it reduces $30 \%-62 \%$ of code and on the consumer side it reduces $9 \%-40 \%$ of the code depending on the amount and complexity of transformations required. 
International Journal of Applied Engineering Research ISSN 0973-4562 Volume 16, Number 5 (2021) pp. 357-361

(c) Research India Publications. https://dx.doi.org/10.37622/IJAER/16.5.2021.357-361

TABLE III

TABLE FOR CASE 3 RESULTS

\begin{tabular}{|c|c|c|c|}
\hline Type of & \multicolumn{3}{|c|}{ Additional Lines of Code } \\
\cline { 2 - 4 } Messaging Service & Service A & Service B & Service $\boldsymbol{C}$ \\
\hline $\begin{array}{l}\text { Asynchronous with Camel } \\
\text { messaging service }\end{array}$ & 21 & 29 & 29 \\
\hline $\begin{array}{l}\text { Asynchronous without Camel } \\
\text { messaging service }\end{array}$ & 21 & 29 & 29 \\
\hline
\end{tabular}

The messaging service fails to provide any benefits, in terms of reducing the addition of lines of code, when there are no transformations required.

But here the service creates a data abstraction layer between consumer and producer service and hides the data schema details of one service from another, hence further decouples the producer and consumer services .

\section{A. Advantages}

The messaging service allows highly scalable and resilient messaging between modules in a microservice. The service can be used without much change to the existing code of the producer or consumer service. This is helpful in allowing service to easily communicate with each other and scale efficiently.

\section{B. Disadvantages}

One of the major drawbacks of this messaging service is that it is not helpful in communication between services when no transformation is required.

It can be used only in applications that use JSON message format. Various other message formats such as Google Protocol Buffer and Apache Avro are used by industries and require support.

Even though the Jolt transformer performs well in simple transformations, it cannot be used to perform complex transformations.

\section{Application}

This messaging service is a generic messaging service that can be used by existing or new producer and consumer services in a microservice architecture to communicate with each other without much changes or addition to the code base of the service.

It is particularly helpful when schema transformations are required for communication and also schema integrity is quintessential.

It can only be used with services that use JSON messaging format.

It is more suitable when the message schema of the producer and consumer service is not the same and transformation of schema is required.

\section{CONCLUSION}

Microservice architecture is in demand due to its scalability, resiliency and autonomous nature. Communication between the services is really important to make this architecture work. But it is also the most complex task. Hence creating a messaging service that leverages a microservice architecture is very necessary.

This paper proposes a lightweight messaging service that can be easily integrated with services and hence helps in building a microservice architecture easily. The service can be integrated with new or existing producer and consumer services with addition of only a few lines of code. It also provides other functionalities such as schema transformation, schema compatibility and integrity validation. Without these functionalities provided by the messaging, the producer or the consumer service would have to accomodate the functionalities, this in turn would increase the lines of code of the services.

Thus, the new messaging service is light weight, highly resilient and easily integrable.

\section{FUTURE WORK}

The ultimate goal is to create a generic messaging service that supports various types of message formats such as Google Protocol Buffer and Apache Avro. Another important functionality that needs to be added is to support schema evolution, which is very important in organisations.

The final goal is to allow other transformations libraries that support complex transformations for not only JSON but also for avro and protobuf.

With these changes a generic messaging service that allows easy integration, high scalability can be built. This messaging service can be used by a wide range of services in a microservice architecture.

\section{REFERENCES}

[1] Baazizi. Mohamed-Amine, Colazzo. Dario, Ghelli. Giorgio Sartiani. Carlo, "Schemas and Types for JSON Data: From Theory to Practice," SIGMOD '19: Proceedings of the 2019 International Conference on Management of Data, pp. 2060-2063, 2019, doi: 10.1145/3299869.3314032.

[2] Dinari. Hamed, "Inter-Process Communication (IPC) in Distributed Environments: An Investigation and Performance Analysis of Some Middleware Technologies," International Journal of Modern Education and Computer Science, pp. 36-52, 2020, doi:10.5815/ijmecs.2020.02.05.

[3] F. Gosewehr, J. Wermann, W. Borsych and A. W. Colombo, "Apache camel based implementation of an industrial middleware solution," 2018 IEEE Industrial Cyber-Physical Systems (ICPS), pp. 523-528, 2018, doi: 10.1109/ICPHYS.2018.8390760.

[4] P. Di Francesco, "Architecting Microservices," 2017 IEEE International Conference on Software Architecture Workshops (ICSAW), pp. 224-229, 2017, doi: 10.1109/ICSAW.2017.65.

[5] Shafabakhsh. Benyamin, Robert. Lagerström and Hacks. Simon, "Evaluating the Impact of Inter Process Communication in Microservice Architectures," QuASoQ 2020 8th International Workshop on Quantitative Approaches to Software Quality, 2020.

[6] Shaheen. Javed, "Apache Kafka: Real Time Implementation with Kafka Architecture Review," International Journal of Advanced Science and Technology, pp. 35-42, 2017, doi: 10.14257/ijast.2017.109.04.

[7] Smid. Antonin, Wang. Ruolin and Cerný. Tom, "Case study on data communication in microservice architecture," RACS, pp. 261-267, 2019, doi: $10.1145 / 3338840.3355659$. 